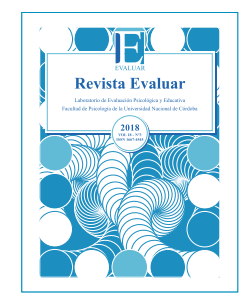

\title{
Propiedades psicométricas de la Escala de Florecimiento en Puerto Rico
}

\section{Psychometric Properties of the Flourishing Scale in Puerto Rico}

\author{
Juan Aníbal González-Rivera * 1 \\ 1 - Ponce Health Sciences University, San Juan University Center, Puerto Rico
}

Introducción
Método
Resultados
Discusión
Referencias

Recibido: 01/03/2018 Revisado: 20/06/2018 Aceptado: 07/07/2018

\section{Resumen}

En el presente estudio se analizan las propiedades psicométricas de la Escala de Florecimiento en Puerto Rico. Se realizaron tres estudios. En el primero, con 665 participantes, se analizaron la confiabilidad y la estructura factorial de la escala. En el segundo, con 821 participantes, se efectuó un análisis factorial confirmatorio. En el tercero, con 342 mujeres puertorriqueñas, se calculó la validez convergente y divergente de las escalas con otras medidas de bienestar y depresión. Los resultados de los estudios mostraron propiedades psicométricas adecuadas en la escala. El índice de confiabilidad Alfa de Cronbach de la escala fue de .92. La varianza explicada en el análisis factorial exploratorio fue de un $65.35 \%$. Los resultados confirmaron que la escala posee una estructura unidimensional. Los ocho ítems de la escala cumplieron con los criterios de discriminación. Se realizaron análisis de validez convergente y discriminante mediante la evaluación de correlación entre el florecimiento, la satisfacción con la vida y la depresión. Se obtuvieron valores estadísticamente significativos. Estos resultados sugieren que la Escala de Florecimiento tiene el potencial para medir este constructo en adultos puertorriqueños.

Palabras clave: bienestar, bienestar psicológico, florecimiento, propiedades psicométricas, validación

\begin{abstract}
This article examines the psychometric properties of the Flourishing Scale in Puerto Rico. Three studies were carried out with this purpose. The first study, of 665 participants, analyzed the reliability and factorial structure of the scales. The second study, of 821 participants, carried out a confirmatory factorial analysis. The third study, of 342 Puerto Rican women, calculated the convergent and divergent validity of the scales with other measures of wellbeing and depression. The results of the studies showed adequate psychometric properties in the scale. The reliability index of the scale was of .95 . The variance in the exploratory factorial analysis was $65.35 \%$. The results confirmed that the scale has a one-dimensional structure. The eight items complied with the criteria of discrimination. Convergent and discriminant validity analyses were performed by evaluating correlation between flourishing, satisfaction with life and depression, obtaining statistically significant values. These results suggest that the Flourishing Scale has the potential to measure this construct among Puerto Rican adults.
\end{abstract}

Key words: flourishing, psychological well-being, psychometric properties, validation, well-being 


\section{Introducción}

Históricamente, la búsqueda de la felicidad y el bienestar humano han sido temas centrales en la literatura, la filosofía y la teología (González-Rivera, Quintero-Giménez, Veray-Alicea, \& Rosario-Rodríguez, 2016). Por ejemplo, en la antigua Grecia, Aristóteles (2001) ya proponía el concepto de la suma felicidad como el fin último del ser humano. En Oriente, los discípulos de Confucio describieron la buena vida (una vida feliz) desde una sociedad ordenada en donde cada ciudadano tiene roles, deberes y responsabilidades claramente delimitados (Diener \& Suh, 2000). Posturas filosóficas como estas inquietaron académicamente a los principales exponentes de la psicología positiva, quienes formalizaron científica y rigurosamente el estudio de la felicidad y el bienestar general en las ciencias sociales (Diener, 2000; Ryff, 1989; Seligman \& Csikszentmihalyi, 2000; Seligman \& Peterson, 2003). La intención principal de estos autores era entender los procesos psicológicos que subyacen en la búsqueda de la felicidad y que fomentan un mayor bienestar en las personas.

Con este propósito, los psicólogos positivos desarrollaron distintos constructos para operacionalizar la felicidad y el bienestar personal y facilitaron su estudio en los campos de la psicología y las ciencias sociales ( $p$. ej. satisfacción con la vida, afecto positivo, afecto negativo, crecimiento y desarrollo personal, entre otros). Estos constructos suelen categorizarse en dos perspectivas distintas de investigación: la hedónica y la eudaemónica. La perspectiva hedónica, caracterizada por el bienestar subjetivo, concibe el bienestar del individuo como la evaluación que las personas hacen sobre la satisfacción que experimentan en sus vidas y el balance entre los afectos positi- vos y negativos (Diener, 2000; Keyes, Shmotkin, \& Ryff, 2002).

Para medir el bienestar desde la perspectiva hedónica, se deben tener en consideración dos aspectos principales: el componente cognitivo -asociado al juicio valorativo-subjetivo que la persona hace sobre su vida- y el componente afectivo-emocional (Diener, 2000; Diener \& Suh, 2000). Tomando como base estos elementos, se han desarrollado varios instrumentos para evaluar el bienestar desde la perspectiva hedónica: el Satisfaction with Life Scale (Diener, Emmons, Larsen, \& Griffin, 1985), el Positive and Negative Affect Schedule (Watson, Clark, \& Tellegen, 1988) y el Scale of Positive and Negative Experience (Diener et al., 2010).

Por su parte, la perspectiva eudaemónica, representada por el bienestar psicológico, asocia el bienestar del individuo con el desarrollo del potencial humano. Los investigadores fieles a esta perspectiva de investigación explican que el bienestar humano está intrínsecamente ligado a la realización de actividades congruentes con los valores profundos del individuo que fomentan su desarrollo y crecimiento personal (Ryan \& Deci, 2001). En síntesis, esta perspectiva concibe la felicidad como un estado de plenitud y armonía en el que el bienestar humano es entendido como la percepción del sentido y propósito en la vida, siendo el crecimiento y desarrollo personal, los principales factores del funcionamiento óptimo del ser humano (Chitgian-Urzúa, Urzúa, \& Vera-Villarroel, 2013; González-Rivera et al., 2016).

Se han desarrollado varios instrumentos para medir el bienestar desde la perspectiva eudaemónica; entre los más conocidos se encuentran: el Questionnaire for Eudaimonic Well-Being (Waterman et al., 2010) y el Scales of Psychological Well-Being (Ryff, 1995). Uno de los últimos instrumentos desarrollados en esta línea de investigación, que destaca por su brevedad y facilidad 
de administración, es la Escala de Florecimiento (Flourishing Scale) de Diener et al. (2010). La Figura 1 presenta gráficamente el desarrollo teórico de los principales instrumentos que examinan el bienestar humano desde las perspectivas hedónica y eudaemónica.

La Escala de Florecimiento fue desarrollada por Diener et al. (2009; 2010) para medir la prosperidad psicosocial, tomando como fundamento conceptual la integración de las principales teorías psicológicas que tratan el florecimiento humano y la prosperidad personal. En primer lugar, en la elaboración de la escala utilizaron las necesidades psicológicas universales conceptua- lizadas por Ryff (1989), estas son: necesidad de competencia, relaciones humanas (interpersonales) y autoaceptación. Asimismo, integraron otros elementos teóricos que, según los autores, fortalecen la perspectiva de las necesidades universales: la noción de capital social (Helliwell, Barrington-Leigh, Harris, \& Huang, 2010), el flow y el compromiso (Csikszentmihalyi, 1990) $\mathrm{y}$, por último, el optimismo, el propósito y el significado (Seligman, 2002). Todos estos elementos psicológicos están contemplados en la Escala de Florecimiento, dado que son beneficiosos para el funcionamiento óptimo del ser humano.

En cuanto a las propiedades psicométricas,

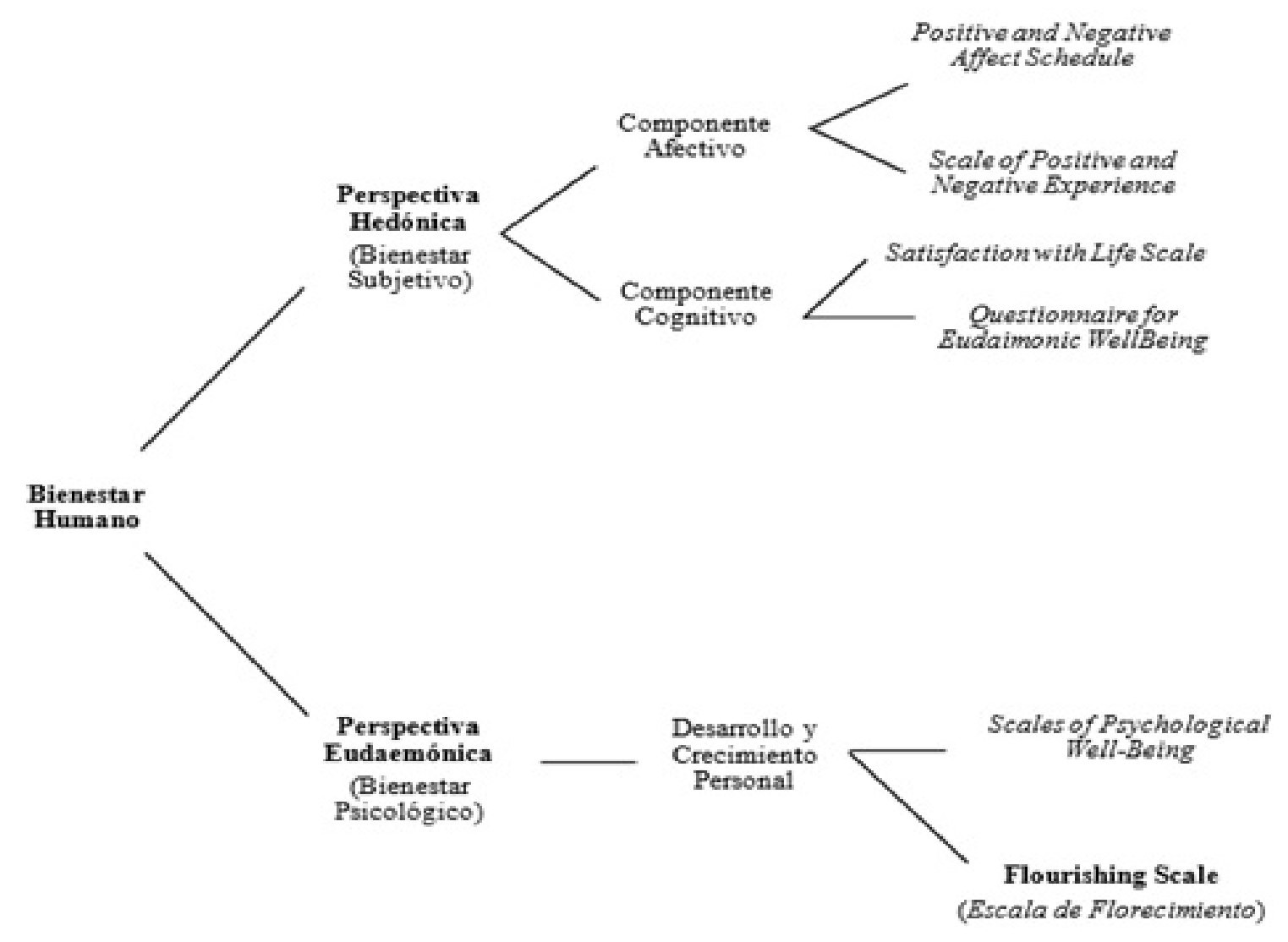

Figura 1

Desarrollo teórico de los principales instrumentos que examinan el bienestar humano desde las perspectivas hedónica y eudaemónica. 
el instrumento ha demostrado una fuerte consistencia interna, con coeficientes alfa de Cronbach que oscilan entre .87 a .95 en diferentes países y culturas (Cassaretto-Bardales \& Martinez-Uribe, 2017; Diener et al., 2010; Esch, Jose, Gimpel, Von Scheidt, \& Michalsen, 2013; Hone, Jarden, \& Schofield, 2014; Howell \& Buro, 2015; Silva \& Caetano, 2013; Sumi, 2013). Por su parte, cuando se validó la Escala de Florecimiento por primera vez en Estados Unidos, se evidenciaron índices de discriminación de los ítems que fluctuaron entre .57 y .71, y la escala reflejó una solución unifactorial que explicaba el $53 \%$ de la varianza de la escala (Diener et al., 2010). Estudios posteriores han confirmado la unidimensionalidad de la escala (Cassaretto-Bardales \& Martinez-Uribe, 2017; Hone et al., 2014; Howell \& Buro, 2015; Silva \& Caetano, 2013).

Si bien la mayoría de los estudios que utilizan la Escala de Florecimiento se han llevado a cabo en Estados Unidos, se han registrado traducciones y validaciones en diferentes contextos internacionales, como Japón (Sumi, 2013), Portugal (Silva \& Caetano, 2013), Canadá (Howell \& Buro, 2015), Nueva Zelanda (Hone et al., 2014), Alemania (Esch et al., 2013) y Perú (Cassaretto-Bardales \& Martinez-Uribe, 2017). Finalmente, cabe señalar que solo existe un estudio que examina las propiedades psicométricas del instrumento en el contexto latinoamericano (Perú), lo que hace necesario incrementar los estudios de carácter psicométricos que examinen la Escala de Florecimiento en otros países hispanohablantes como Puerto Rico.

El presente estudio se propuso verificar las propiedades psicométricas de la Escala de Florecimiento en la población puertorriqueña y comprobar si es un instrumento adecuado para examinar el bienestar psicológico en adultos puertorriqueños. En este sentido, nuestro estudio pretende validar y analizar las propiedades psicométricas de la versión en español de la Escala de Florecimiento en adultos puertorriqueños. Para ello, se llevaron a cabo tres estudios independientes. En el Estudio 1 se analiza la estructura factorial de la escala mediante un análisis factorial exploratorio, se evalúa la confiabilidad del instrumento y se examina la capacidad de discriminación de los ítems. En el Estudio 2 se analiza la validez de constructo mediante un análisis factorial confirmatorio con ecuaciones estructurales y se evalúa la confiabilidad compuesta. En el Estudio 3 se evalúa la validez convergente y divergente de la escala con respecto a otras medidas de bienestar y una escala de depresión. En los tres estudios se utiliza un diseño de investigación instrumental (Montero \& León, 2007).

\section{Estudio 1}

El objetivo de este estudio fue analizar la estructura factorial de la escala mediante un AFE de componentes principales, analizar los índices de discriminación de los ocho ítems y evaluar la confiabilidad de la escala mediante dos métodos: coeficiente alfa de Cronbach y división en mitades de Spearman-Brown.

\section{Método}

Participantes. Se trabajó con una muestra no probabilística constituida por 665 participantes: 473 mujeres (71.1\%) y 192 hombres $(28.9 \%)$. El rango de edad fue de entre los 21 y 65 años, con una edad promedio de $35.86(\mathrm{DE}=10.22)$. En cuanto al estado civil, el 26.2\% $(\mathrm{n}=174)$ era soltero, el $44.8 \%(n=298)$ era casado y el $29 \% n=193)$ convivía con su pareja sin nupcias. En términos de preparación académica, el 40\% $(\mathrm{n}=266)$ poseía un bachillerato, el $24.5 \%(\mathrm{n}=163)$ poseía 
un grado de maestría, el $18.8 \%(\mathrm{n}=125)$ tenía un grado técnico o grado asociado, el $6 \%(\mathrm{n}=$ 40) completó únicamente la escuela superior, y el $10.7 \%(n=71)$ contaba con un doctorado. Por último, la mayoría de la muestra (el 51.1\%, $\mathrm{n}=340)$ generaba ingresos menores que $\$ 25000$, mientras que el $32.6 \%(n=217)$ entre $\$ 26000$ y $\$ 50000$.

Instrumentos. La Escala de Florecimiento (Diener et al., 2010) está constituida por ocho ítems que examinan el bienestar psicológico desde una perspectiva eudaemónica. Cada ítem posee una escala de respuesta de siete puntos, cuyos límites lo constituyen las respuestas Muy en desacuerdo (1) y Muy de acuerdo (7). El rango posible es de 8 a 56 puntos donde a mayor puntuación, mayor grado de crecimiento o desarrollo psicológico.

Procedimientos generales. La recopilación de datos se llevó a cabo mediante el uso de cuestionarios a través de la plataforma PsychData utilizando como método de reclutamiento las redes sociales: Facebook, Twitter, Google+, WhatsApp, entre otras. Esta investigación fue aprobada por el Comité para la Ética en la Investigación (IRB) de la Universidad Carlos Albizu en San Juan, Puerto Rico. Los datos fueron analizados en el programa estadístico IBM SPSS versión 24.0 (IBM Corporation, 2016).

\section{Resultados}

Análisis de componentes principales. En el primer estudio se realizó un análisis AFE utilizando el método de extracción de componentes principales con rotación oblicua para identificar las variables latentes que subyacen en los ítems. Los resultados mostraron una estructura unifactorial con un valor característico de 5.23, que explica el $65.35 \%$ de la varianza de los datos $(\mathrm{KMO}=.92$; $\left.\chi^{2}=3511.974_{(28)}, p<.001\right)$. Las cargas factoriales de los ítems sobrepasan el .72, superando el mínimo de .50 recomendado por Stevens (2002). En la Tabla 1 se presentan las cargas factoriales obtenidas por los 8 ítems.

Análisis de Discriminación. Una vez que se analizó la estructura factorial de la escala, se llevó a cabo un análisis de reactivos para calcular los índices de discriminación de los ocho ítems de

\section{Tabla 1}

Análisis factorial exploratorio e índices de discriminación de los ítems.

\begin{tabular}{lll}
\hline \multicolumn{1}{c}{ Ítems } & $r_{b i s}$ & \multicolumn{1}{c}{$\mathbf{1}$} \\
\hline 1. Llevo una vida significativa y con propósito. & .78 & .84 \\
2. Mis relaciones sociales me apoyan y son reconfortantes. & .69 & .76 \\
3. Me intereso y me involucro en mis actividades diarias. & .79 & .85 \\
4. Contribuyo activamente a la felicidad y al bienestar de otros. & .74 & .81 \\
5. Soy competente y capaz en las actividades que son importantes para mí. & .74 & .82 \\
6. Soy una buena persona y vivo una buena vida. & .79 & .86 \\
7. Soy optimista acerca de mi futuro. & .75 & .81 \\
8. La gente me respeta. & .64 & .72 \\
\hline
\end{tabular}

Nota. $r_{b i s}=$ índice de discriminación del ítem; 1 = cargas factoriales $(\mathrm{n}=665)$. 
la Escala de Florecimiento, mediante el método de correlación ítem-total de la prueba $\left(r_{\text {bis }}\right)$. Los resultados mostraron índices de discriminación que fluctuaban entre .64 y .79, de manera que todos los reactivos obtuvieron índices de discriminación mayores a .30, como recomienda Kline (2005). La Tabla 1 presenta los índices de discriminación de los ocho ítems de la escala.

Análisis de Confiabilidad.Los ocho ítems fueron sometidos a un análisis de consistencia interna para determinar el índice de confiabilidad de la escala. Para ello utilizamos dos métodos: el coeficiente Alfa de Cronbach y división en mitades de Spearman-Brown. Los resultados mostraron un coeficiente alfa de .92 y de .92 para la prueba Spearman-Brown. La Tabla 2 presenta el alfa de Cronbach, alfa de Cronbach estandarizado, el coeficiente de la prueba Spearman-Brown, la media y la desviación estándar de la Escala de Florecimiento.

\section{Estudio 2}

Los objetivos de este segundo estudio fueron analizar la validez de constructo mediante un AFC con ecuaciones estructurales y evaluar la fiabilidad compuesta del instrumento.

\section{Método}

Participantes. Para este estudio se reclutó una muestra no probabilística constituida por 821 participantes: 410 mujeres (49.9\%) y 411 hombres (50.1\%). El rango de edad fue de entre los $19 \mathrm{y}$ 75 años, con una edad promedio de 37.24 (DE = 13.30). En cuanto al estado civil, el 50.7\% ( $\mathrm{n}=$ $417)$ era soltero, el $33.6 \%(n=275)$ era casado y el $15.7 \%(n=129)$ convivía con su pareja sin nupcias. En términos de preparación académica, el 49\% $(n=402)$ poseía un bachillerato, el 17.4\% $(\mathrm{n}=143)$ poseía un grado de maestría, el 16.9\% $(\mathrm{n}=139)$ tenía un grado técnico o grado asociado, el 8.9\% $(\mathrm{n}=73)$ cursó únicamente la escuela superior, y el 7.8\% $(\mathrm{n}=64)$ contaba con un doctorado. Por último, la mayoría de la muestra (el $58.2 \%, \mathrm{n}=478)$ generaba ingresos menores que $\$ 25000$, mientras que el $29.4 \%(n=241)$ entre $\$ 26000$ y $\$ 50000$.

Instrumento. En el Estudio 2 solo se utilizó la Escala de Florecimiento (véase descripción en el

\section{Tabla 2}

Medias, desviaciones estándar y coeficientes de confiabilidad.

\begin{tabular}{|c|c|c|c|c|c|}
\hline Factor & M & $\mathbf{D E}$ & $\alpha$ & $\alpha_{e s t}$ & $\begin{array}{c}\text { Spearman- } \\
\text { Brown }\end{array}$ \\
\hline Escala de Florecimiento & 47.68 & 8.89 & .92 & .92 & .90 \\
\hline
\end{tabular}

Nota. $\mathrm{M}=$ media; $\mathrm{DE}=$ desviación estándar; $\alpha=$ alfa de Cronbach; $\alpha_{\text {est }}=$ alfa de Cronbach estandarizado. $(\mathrm{n}=665)$. 
Estudio 1) con el propósito de auscultar sus características psicométricas.

Procedimientos generales. La recopilación de datos se llevó a cabo de forma electrónica y presencial durante el 2017. Se reclutaron personas de forma presencial $(n=348)$ en dos centros universitarios de la zona metropolitana de Puerto Rico y en varias oficinas de empleo. A su vez, se reclutaron participantes electrónicamente $(n=473)$ mediante un anuncio pagado en Facebook y otras redes sociales. Esta investigación fue aprobada por el Comité para la Ética en la Investigación IRB) de la Universidad Carlos Albizu en San Juan, Puerto Rico. Los programas estadísticos utilizados en esta fase fueron IBM SPSS Statistics versión 24 y STATA versión 14.1 (StataCorp., 2017).

\section{Resultados}

Análisis descriptivos de los ítems. En primer lugar, se calcularon las medias y las desviaciones estándar para cada ítem de la escala para analizar las propiedades de distribución de la escala. Las medias de los ítems fluctuaron entre $6.03 \mathrm{y}$ 6.57, con desviaciones estándar que fluctuaron entre .95 y 1.22. Como muestra, la Tabla 3 y las pruebas de normalidad de Kolmogorov-Smirnov y Shapiro-Wilk evidencian que los datos no se distribuyen de forma normal $(p<.001)$. Por la falta de normalidad en los datos, utilizamos las correcciones de Satorra y Bentler (2001) para calcular el ajuste de los modelos de ecuaciones estructurales, ya que la no normalidad de los datos altera los errores de estimación y el ajuste global del modelo.

Validez de Constructo. Se realizó un AFC con ecuaciones estructurales utilizando el método de estimación de máxima verosimilitud. Para evaluar el ajuste del modelo se utilizaron los siguientes índices de bondad de ajuste y los criterios de Byrne (2010): chi-cuadrado $\left(\chi^{2}\right)$, error medio cuadrático de aproximación (RMSEA; los valores sugeridos deben ser $\leq .08$ para indicar un buen ajuste), índice Tucker-Lewis (TLI) y el índice de ajuste comparativo (CFI). Para que exista un buen ajuste del modelo, los valores sugeridos de CFI y TLI deben ser de $\geq .95$ (Byrne, 2010). Los resultados arrojaron un buen ajuste para el modelo conformado por un solo factor latente que recogía los ocho ítems de la escala (véase Figura 2), $\chi^{2}=181.315_{(20)} p<.001, \mathrm{RMSEA}=.08$, $\mathrm{CFI}=.97, \mathrm{TLI}=.96, \chi^{2}$ corregido $=81.755_{(20)} p$ $<.001$, RMSEA corregido $=.05$, CFI corregido $=$ .97 , TLI corregido $=.96$. Estos índices cumplen con lo que Satorra y Blenter (2001) consideran como niveles aceptables. A su vez, se examinaron los coeficientes de regresión de cada ítem, esperando que cada uno de estos fuese mayor a .65 , según recomiendan Schumaker y Lomax (2010). Los coeficientes de regresión fluctuaron entre .68 y .85 (véase Tabla 4). Luego, se analizó la confiabilidad compuesta del modelo unidimensional de la escala. Los análisis revelaron un índice de confiabilidad compuesta de .93, lo que supera el valor mínimo recomendado por la literatura (Raykov \& Shrout, 2002).

Diferencias entre hombres y mujeres. Se realizó una prueba $t$ independiente para analizar si existen diferencias estadísticamente significativas entre las medias de florecimiento de las mujeres (M $=49.91, \mathrm{DE}=7.98) \mathrm{y}$ de los hombres $(\mathrm{M}=50.81$, $\mathrm{DE}=6.11)$. Los resultados confirmaron que la diferencia a favor de los hombres no es estadísticamente significativa $\left(t_{(819)}=-1.815, p>.05\right)$. 
Tabla 3

Estadísticos de descriptivos y de distribución para los ítems de la escala.

\begin{tabular}{ccccccc}
\hline Ítem & Media & $\begin{array}{c}\text { Desviación } \\
\text { Estándar }\end{array}$ & Asimetría & Curtosis & $\begin{array}{c}\text { Kolmogorov- } \\
\text { Smirnov }\end{array}$ & Shapiro-Wilk \\
\hline 1 & 6.33 & 1.14 & -2.59 & 7.94 & .32 & .62 \\
2 & 6.03 & 1.19 & -1.74 & 3.55 & .28 & .76 \\
3 & 6.29 & 1.07 & -2.45 & 7.62 & .29 & .66 \\
4 & 6.34 & 1.05 & -2.50 & 7.99 & .32 & .64 \\
5 & 6.57 & .95 & -3.70 & 16.68 & .29 & .49 \\
6 & 6.49 & .97 & -3.20 & 13.17 & .36 & .57 \\
7 & 6.20 & 1.19 & -2.01 & 4.40 & .29 & .70 \\
8 & 6.11 & 1.22 & -1.94 & 4.24 & .27 & .72
\end{tabular}

Nota. Error estándar de la asimetría $=.09$; Error estándar de la curtosis $=.17$. Grados de libertad Kolmogorov-Smirnov y Shapiro-Wilk $=821$, todos los valores $p<.001$.

\section{Estudio 3}

Una vez confirmada la confiabilidad de la escala, su estructura factorial y que las puntuaciones de la escala no se alteran según el género, el objetivo de este tercer estudio fue determinar la validez convergente y divergente de las escalas con otras medidas de bienestar y una medida de sintomatología depresiva.

\section{Método}

Participantes. Este estudio contó con la participación de 342 mujeres adultas puertorriqueñas, seleccionadas por disponibilidad. El rango de edad fue de entre los 21 y 68 años, con una edad promedio de $37.66(\mathrm{DE}=10.73)$. En términos de preparación académica, el 38.3\% $(\mathrm{n}=131)$ poseía un bachillerato, el $26.9 \%(\mathrm{n}=92)$ poseía un grado de maestría, el $20.7 \%(\mathrm{n}=71)$ tenía un grado técnico o grado asociado, el 5\% $(\mathrm{n}=17)$ completó únicamente la escuela superior, y el 9.1\% $(\mathrm{n}=31)$ contaba con un doctorado. Por último, la mayoría de la muestra (el 44.7\%, $\mathrm{n}=153$ ) generaba ingre-

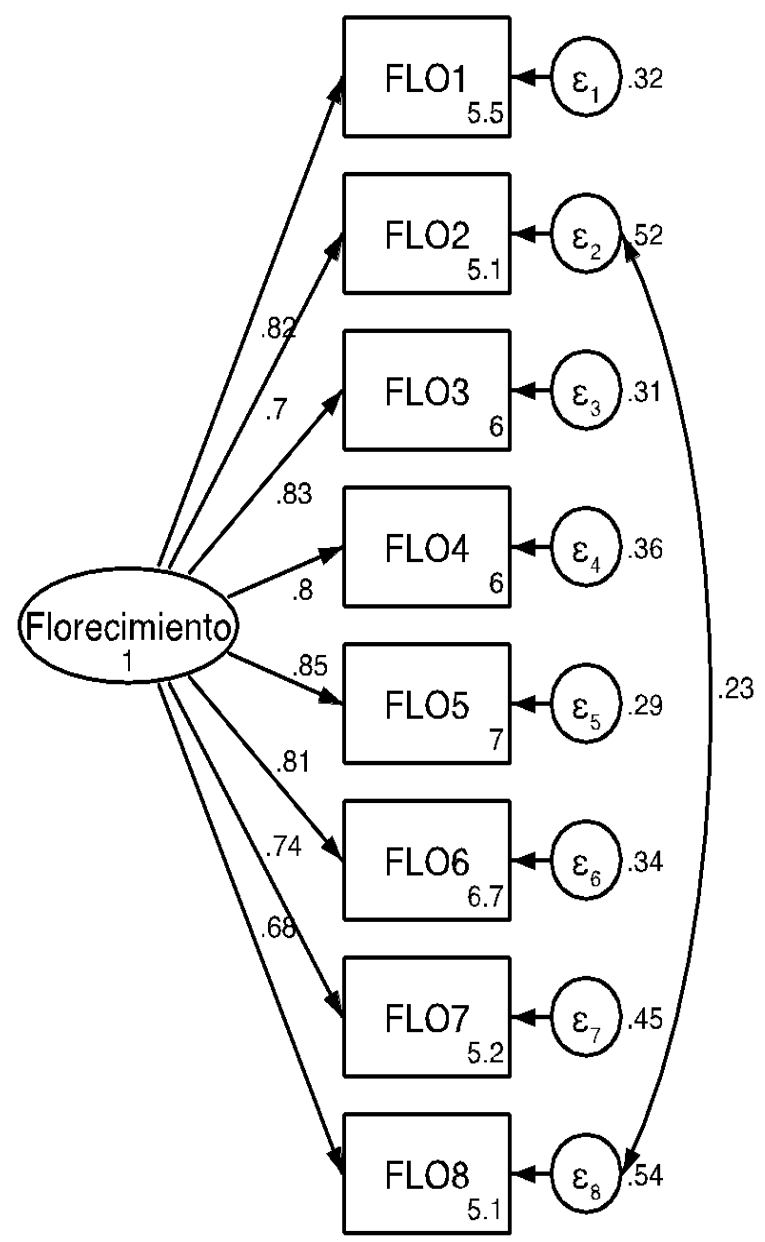

Figura 2

Modelo unidimensional de la Escala de Florecimiento. 
Tabla 4

Estimados estandarizados de regresión y sus intervalos de confianza.

\begin{tabular}{llll}
\hline \multicolumn{1}{c}{ Ítems } & $\boldsymbol{\beta}$ & $\boldsymbol{p}$ & $\mathbf{I . C . 9 5 \%}$ \\
\hline 1. Llevo una vida significativa y con propósito. & .82 & $<.001$ & {$[.77, .87]$} \\
2. Mis relaciones sociales me apoyan y son reconfortantes. & .70 & $<.001$ & {$[.63, .76]$} \\
3. Me intereso y me involucro en mis actividades diarias. & .83 & $<.001$ & {$[.79, .88]$} \\
4. Contribuyo activamente a la felicidad y al bienestar de otros. & .80 & $<.001$ & {$[.74, .86]$} \\
5. Soy competente y capaz en las actividades que son importantes para mí. & .85 & $<.001$ & {$[.80, .90]$} \\
6. Soy una buena persona y vivo una buena vida. & .81 & $<.001$ & {$[.75, .88]$} \\
7. Soy optimista acerca de mi futuro. & .74 & $<.001$ & {$[.69, .79]$} \\
8. La gente me respeta. & .68 & $<.001$ & {$[.60, .75]$} \\
\hline
\end{tabular}

Nota. $\beta=$ estimados estandarizados de regresión; $p=$ significación; I.C. $95 \%=$ intervalos de confianza de los coeficientes de regresión.

sos menores que $\$ 25000$, mientras que el $35.7 \%$ $(\mathrm{n}=122)$ entre $\$ 26000$ y $\$ 50000$.

Instrumentos. En el Estudio 3, además de utilizar la Escala de Florecimiento (véase descripción en el Estudio 1), se utilizaron la Escala de Satisfacción con la Vida (Diener et. al., 1985), la Escala de Autoestima de Rosenberg (1965) y la Escala de Depresión del DASS-21 (Lovibond \& Lovibond, 1995). La Escala de Satisfacción con la Vida está constituida por un total de cinco ítems (p. ej. Estoy satisfecho/a con mi vida) con una escala de respuesta de siete puntos que fluctúan de Muy en desacuerdo (1) a Muy de acuerdo (7). El puntaje más bajo que se puede obtener es 5 y el más alto es 35, donde puntuaciones elevadas sugieren alta presencia de satisfacción con la vida $(\alpha=.92)$.

Por su parte, la Escala de Autoestima de Rosenberg está constituida por 10 ítems (p. ej. En general, estoy satisfecha conmigo misma) con una escala de respuesta de cuatro puntos que fluctúan de Totalmente de acuerdo (1) a Totalmente en desacuerdo (4). El rango posible es de 10 a 40 puntos, donde elevadas puntuaciones sugieren una alta valía personal y mucho respeto a sí misma $(\alpha=.90)$. Por último, la Escala de Depresión del DASS-21 está constituida por siete ítems ( $\mathrm{p}$. ej. Me sentí triste y deprimido) con una escala de respuesta de cuatro puntos que fluctúan de Nada (0) a Mayor parte del tiempo (3). El puntaje total se calcula con la suma de los ítems pertenecientes a esa escala y varía entre 0 y 21 puntos; a mayor puntuación, mayor sintomatología presenta la participante $(\alpha=.90)$.

Procedimientos generales. La recopilación de datos se llevó a cabo mediante el uso de cuestionarios a través de la plataforma PsychData utilizando como método de reclutamiento las redes sociales: Facebook, Twitter, Google+, WhatsApp, entre otras. Esta investigación fue aprobada por el Comité para la Ética en la Investigación (IRB) de la Universidad Carlos Albizu en San Juan, Puerto Rico. Los datos fueron analizados en el programa estadístico IBM SPSS (versión 24).

\section{Resultados}

Evidencias de Validez Convergente y Divergente. Con el fin de evaluar la validez convergente y divergente de la Escala de Florecimiento, se 
analizaron las correlaciones con otras medidas de bienestar (satisfacción con la vida y autoestima) y una medida de sintomatología asociada con la depresión. Como se puede observar en la Tabla 5, todas las correlaciones fueron significativas. Según lo esperado, las correlaciones entre florecimiento y las otras medidas de bienestar (satisfacción con la vida y autoestima) fueron positivas. En cambio, la correlación entre florecimiento $\mathrm{y}$ depresión fue negativa.

\section{Discusión}

El presente proyecto de investigación tuvo como propósito analizar las propiedades psicométricas de la Escala de Florecimiento de Diener et al. (2010) en una muestra de adultos en Puerto Rico. A partir de los resultados obtenidos, se puede establecer que la escala posee adecuadas características psicométricas para examinar el bienestar humano desde una perspectiva eudaemónica. Específicamente, la escala evalúa relaciones interpersonales positivas, sentimientos de competencia, sentido de propósito y optimismo. Además, los índices de confiabilidad obtenidos sugieren, según lo establece Kline (2000), que la Escala de Florecimiento cuenta con la suficiente consistencia interna para ser utilizada como instrumento de medición científica en investigacio- nes en Puerto Rico y América Latina.

De forma general, el adicionamiento del AFE del Estudio 1 con el AFC del Estudio 2 demuestra que el modelo hipotetizado presenta un ajuste satisfactorio con los datos y confirma la estructura unifactorial del instrumento. Esto es congruente con investigaciones realizadas en otros países que aportan evidencia a la unidimensionalidad de la escala (Cassaretto-Bardales \& Martínez-Uribe, 2017; Hone et al., 2014; Howell \& Buro, 2015; Silva \& Caetano, 2013). Según el AFE, la estructura factorial de la escala explica un $65 \%$ de la varianza total, lo que concuerda con los parámetros de varianza explicados en otras investigaciones internacionales que oscilan entre $47 \%$ y 73\% (Cassaretto-Bardales \& Martínez-Uribe, 2017; Diener et al., 2010; Hone et al., 2014; Silva \& Caetano, 2013; Sumi, 2013).

En cuanto a la confiabilidad del instrumento, se obtuvieron índices superiores a los evidenciados por la escala en la mayoría de los estudios (Cassaretto-Bardales \& Martínez-Uribe, 2017; Diener et al., 2010; Hone et al., 2014; Silva \& Caetano, 2013), únicamente superados por el índice de consistencia interna obtenido en la validación de la escala en Japón (Sumi, 2013). Las correlaciones de cada ítem con la puntuación total manifiestan una consistencia interna notable y superan el rango de .61 - .71 informado en la literatura científica (Cassaretto-Bardales \& Mar-

\section{Tabla 5}

Correlaciones entre las escalas, sus medias, desviaciones estándar y Alfa de Cronbach.

\begin{tabular}{|c|c|c|c|c|c|c|}
\hline & $\alpha$ & $\mathbf{M}$ & DE & 1 & 2 & 3 \\
\hline 1. Florecimiento & .92 & 44.85 & 10.08 & & & \\
\hline 2. Satisfacción con la Vida & .92 & 22.76 & 8.01 & .73 & & \\
\hline 3. Autoestima & .90 & 33.49 & 6.18 & .50 & .50 & \\
\hline 4. Depresión & .90 & 5.77 & 5.23 & -.47 & -50 & -51 \\
\hline
\end{tabular}

Nota. Todas las correlaciones significativas a .01. $(\mathrm{n}=342) ; \alpha=$ Alfa de Cronbach 
tínez-Uribe, 2017; Diener et al., 2010).

Por su parte, la correlación moderadamente alta de la Escala de Florecimiento con la medida de satisfacción con la vida aporta evidencia sobre la validez convergente de la escala y concuerda con los patrones de correlaciones obtenidos en otras investigaciones, tanto en Puerto Rico como internacionalmente (González-Rivera, Pabellón-Lebrón, \& Rosario-Rodríguez, 2017; Hone et al., 2014; Silva \& Caetano, 2013). Esto demuestra que la Escala de Florecimiento es una medida confiable de bienestar eudaemónico y distinguible de las medidas de bienestar hedónico. Además, la relación obtenida con la presencia de sintomatología depresiva también es la esperada y la observada en estudios previos (Faulk, Gloria, \& Steinhardt, 2013; Keyes, 2002; Keyes, 2006), lo que confirma que la escala posee validez discriminante.

En términos de las implicaciones teóricas del estudio, los hallazgos psicométricos demostraron que la escala está organizada de forma coherente con lo planteado por sus autores. A su vez, las similitudes de los resultados en todas las validaciones de la escala en distintos países sugieren que la escala no se ve afectada por variables y atributos culturales. Este detalle permite suponer cierta consistencia teórica sobre los procesos psicológicos que facilitan y desarrollan el florecimiento y el bienestar humano. En este sentido, nuestros resultados robustecen los fundamentos teóricos de la psicología del bienestar y la psicología positiva. Además, como señalan Diener et al. (2010) y Cassaretto-Bardales y Martínez-Uribe (2017), se confirmó con los análisis descriptivos la tendencia a experimentar altos niveles de bienestar, independientemente de la muestra que se utilice a nivel internacional.

En cuanto a las implicaciones prácticas del estudio, se demostró que la Escala de Florecimiento es útil en el desarrollo de nuevas investi- gaciones en los campos de la psicología positiva y de la psicología de la salud en Puerto Rico y América Latina (Cassaretto-Bardales \& Martínez-Uribe, 2017). En términos de conveniencia, las principales fortalezas de la escala son su lenguaje sencillo y su brevedad, lo que la consolida como un instrumento de fácil administración e interpretación. En este sentido, la Escala de Florecimiento se constituye como una herramienta práctica y efectiva en el quehacer investigativo y académico de la psicología latinoamericana.

Como parte de las limitaciones de esta investigación, se encuentra el tipo de muestreo no probabilístico por disponibilidad. Esto hace que la generalización de los resultados sea limitada. Sin embargo, se obtuvo una muestra heterogénea y de un tamaño razonable en los tres estudios para poder examinar las propiedades psicométricas del instrumento en tres muestras independientes. Además, el uso de una plataforma electrónica es una limitación porque limita la oportunidad de participación solo a personas que tengan acceso a internet, aún cuando se trata de una técnica en auge por facilitar una recolección ágil.

Para futuros estudios, se recomienda examinar la confiabilidad temporal a través de la técnica test-retest. A su vez, deben estudiarse las propiedades psicométricas de la escala en otras poblaciones como, por ejemplo, adolescentes y pacientes psiquiátricos. De igual forma, se deben llevar a cabo análisis de comparación según las variables demográficas, particularmente edad, estatus socioeconómico y preparación académica, para identificar diferencias entre los grupos. Por último, se sugiere replicar estos resultados en muestras más amplias y/o equilibradas por sexo.

\section{Conclusiones generales}

En síntesis, los resultados obtenidos en 
cuanto a validez y confiabilidad indican que las propiedades psicométricas de la Escala de Florecimiento son adecuadas. El hecho de utilizar tres muestras independientes para validar y examinar la escala en Puerto Rico da un valor añadido al estudio. Se concluye que la Escala de Florecimiento es un excelente instrumento de medición para futuras investigaciones en Puerto Rico, así como para la práctica profesional de la psicoterapia y la consejería. Por último, existe suficiente evidencia psicométrica para considerar la Escala de Florecimiento como un índice transnacional para realizar estudios comparativos entre distintas culturas.

\section{Referencias}

Aristóteles (2001). Ética. Madrid, España: Libsa.

Byrne, B. M. (2010). Structural equation modeling with AMOS: Basic concepts, applications, and programming. New York, NY: Psychology Press.

Cassaretto-Bardales, M., \& Martinez-Uribe, P. (2017). Validación de las escalas de bienestar, de florecimiento y afectividad. Pensamiento Psicológico, 15(1), 19-31.

Chitgian-Urzúa, V., Urzúa, A., \& Vera-Villarroel, P. (2013). Análisis preliminar de las Escalas de Bienestar Psicológico en población chilena. Revista Argentina de Clínica Psicológica, 22(1), 5-14.

Csikszentmihalyi, M. (1990). Flow: The psychology of optimal experience. New York, NY: Harper \& Row.

Diener, E. (2000). Subjective well-being: The science of happiness and proposal for a national index. American Psychologist, 55(1), 34-43. doi: 10.1037/0003066X.55.1.34

Diener, E., \& Suh, E. (2000). Culture and subjective well-being. Cambridge: Massachusetts Institute of Technology.

Diener, E., Emmons, R. A., Larsen, R. J., \& Griffin, S. (1985). The Satisfaction with Life Scale. Journal of Personality Assessment, 49(1), 71-75. doi: 10.1207/ s15327752jpa4901_13
Diener, E., Wirtz, D., Biswas-Diener, R., Tov, W., Kim-Prieto, C., Choi, D.-w., \& Oishi, S. (2009). New measures of well-being. En E. Diener (Ed.), Social indicators research series: Vol. 39. Assessing well-being: The collected works of Ed Diener (pp. 247-266). New York, NY: Springer Science + Business Media. doi: 10.1007/978-90-481-2354-4_12

Diener, E., Wirtz, D., Tov, W., Kim-Prieto, C., Choi, D., Oishi, S., \& Biswas-Diener, R. (2010). New well-being measures: Short scales to assess flourishing and positive and negative feelings. Social Indicators Research, 97(2), 143-156.

Esch, T., Jose, G., Gimpel, C., Von Scheidt, C., \& Michalsen, A. (2013). Die Flourishing Scale (FS) von Diener et al. liegt jetzt in einer autorisierten deutschen Fassung (FS-D) vor: Einsatz bei einer Mind-Body-medizinischen Fragestellung. Forschende Komplementarmedizin, 20(4), 267-275. doi: 10.1159/000354414

Faulk, K. E., Gloria, C. T., \& Steinhardt, M. A. (2013). Coping profiles characterize individual flourishing, languishing, and depression. Anxiety, Stress, \& Coping: An International Journal, 26(4), 378-390. doi: 10.1080/10615806.2012.708736

González-Rivera, J. A., Pabellón-Lebrón, S., \& Rosario-Rodríguez, A. (2017). El rol mediador de la identificación ateísta en la relación entre discriminación y bienestar psicológico: Un estudio preliminar. Revista Puertorriqueña de Psicología, 28(2), 406-421. Recuperado de http://www.ojs.repsasppr.net/index.php/ reps/index

González-Rivera, J. A., Quintero-Giménez, N., Veray-Alicea, J., \& Rosario-Rodríguez, A. (2016). Adaptación y validación de la escala de bienestar psicológico de Ryff en una muestra de adultos puertorriqueños. $\mathrm{Sa}$ lud y Conducta Humana, 3(1), 1-14. Recuperado de http://rsych.com/

Helliwell, J. F., Barrington-Leigh, C., Harris, A., \& Huang, H. (2010). International evidence on the social context of well-being. En E. Diener, D. Kahneman, \& J. F. Helliwell (Eds.), International differences in well-being, (pp. 291-327). Oxford, UK: 
Oxford University Press. doi: 10.1093/acprof:oso/9780199732739.003.0010

Hone, L., Jarden, A., \& Schofield, G. (2014). Psychometric properties of the Flourishing Scale in a New Zealand sample. Social Indicators Research, 119(2), 10311045. doi: 10.1007/s11205-013-0501-X

Howell, A. J., \& Buro, K. (2015). Measuring and predicting student well-being: Further evidence in support of the Flourishing Scale and the Scale of Positive and Negative Experiences. Social Indicators Research, 121(3), 903-915. doi: 10.1007/s11205-014-0663-1

IBM Corporation (2016). IBM SPSS Statistics for Windows, Version 24.0 [Software de computación]. Armonk, NY: IBM.

Keyes, C. L. M. (2002). The mental health continuum: From languishing to flourishing in life. Journal of Health and Social Behavior, 43(2), 207-222. doi: $10.2307 / 3090197$

Keyes, C. L. M. (2006). Mental health in adolescence: Is America's youth flourishing? American Journal of Orthopsychiatry, 76(3), 395-402. doi: 10.1037/00029432.76.3.395

Keyes, C. L. M., Shmotkin, D., \& Ryff, C. D. (2002). Optimizing well-being: The empirical encounter of two traditions. Journal of Personality and Social Psychology, 82(6), 1007-1022. doi: 10.1037/00223514.82.6.1007

Kline, P. (2000). The Handbook of Psychometric Testing. New York: Routledge.

Kline, T. J. B. (2005). Psychological testing: A practical approach to design and evaluation. Thousand Oaks: Sage. doi: 10.4135/9781483385693

Lovibond, S. H., \& Lovibond, P. F. (1995). Manual for the Depression Anxiety Stress Scales (2a Ed.). Sydney: Psychology Foundation.

Montero, I., \& León, O. G. (2007). A guide for naming research studies in psychology. International Journal of Clinical and Health Psychology, 7(3), 847-862. Recuperado de http://www.aepc.es/ijchp/busca. php?coid $=$ Espa $\% \mathrm{~F} 1 \mathrm{ol}$

Raykov, T., \& Shrout, P. E. (2002). Reliability of scales with general structure: Point and interval estimation using a structural equation modeling approach. Structural equation modeling: A Multidisciplinary Journal, 9(2), 195-212. doi: 10.1207/S15328007SEM0902_3

Rosenberg, M. (1965). Society and the adolescent self-image. Princeton, NJ: Princeton University Press. doi: $10.1515 / 9781400876136$

Ryan, R., \& Deci, E. (2001). On happiness and human potentials: A review of research on hedonic and eudaimonic well-being. Annual Review of Psychology, 52(1), 141-166. doi: 10.1146/annurev.psych.52.1.141

Ryff, C. (1989). Happiness is everything, or is it? Exploration on the meaning of psychological well-being. Journal of Personality and Social Psychology, 57(6), 1069-1081. doi: 10.1037/0022-3514.57.6.1069

Ryff, C. (1995). Psychological well-being in adult life. $\mathrm{Cu}$ rrent Directions in Psychological Science, 4(4), 99104. doi: 10.1111/1467-8721.ep10772395

Satorra, A., \& Bentler, P. M. (2001). A scaled difference chi-square test statistic for moment structure analysis. Psychometrika, 66(4), 507-514. doi: 10.1007/ BF02296192

Schumacker, R. E., \& Lomax, R. G. (2010). A beginner's guide to structural equation modeling (3a ed.). Mahwah, NJ: Erlbaum. doi: 10.4324/9780203851319

Seligman, M. E. (2002). Authentic happiness: Using the new positive psychology to realize your potential for lasting fulfillment. New York, NY: Free Press.

Seligman, M. E., \& Csikszentmihalyi, M. (2000). Positive psychology: An introduction. American Psychologist, 55(1), 5-14. doi: 10.1037/0003-066X.55.1.5

Seligman, M. E., \& Peterson, C. (2003). Positive clinical psychology. En L. G. Aspinwall \& U.M. Staudinger (Eds.). A Psychology of human strengths: Fundamental questions and future directions for a positive psychology (pp. 305-317). Washington, DC: American Psychological Association.

Silva, A. J., \& Caetano, A. (2013). Validation of the Flourishing Scale and Scale of Positive and Negative Experience in Portugal. Social Indicators Research, 110(2), 469-478. doi: 10.1007/s11205-011-9938-y 
StataCorp. (2017). Stata: Release 15 [Software de computación]. College Station, TX: StataCorp LLC.

Stevens, J. (2002). Applied multivariate statistics for the social sciences (4a ed.). Mahwah, NJ: Lawrence Erlbaum Associates.

Sumi, K. (2013). Reliability and validity of Japanese versions of the Flourishing Scale and the Scale of Positive and Negative Experience. Social Indicators Research, 118(2), 601-615. doi: 10.1007/s11205013-0432-6

Waterman, A. S., Schwartz, S. J., Zamboanga, B. L., Ravert, R. D., Williams, M. K., Agocha, V. B., . . . Donnellan, M. B. (2010). The Questionnaire for Eudaimonic Well-Being: Psychometric properties, demographic comparisons, and evidence of validity. The Journal of Positive Psychology, 5(1), 41-61. doi: 10.1080/17439760903435208

Watson, D., Clark, L. A., \& Tellegen, A. (1988). Development and validation of brief measures of positive and negative affect: The PANAS scales. Journal of Personality and Social Psychology, 54(6), 1063-1070. doi: 10.1037/0022-3514.54.6.1063 